\section{Monte Carlo Study of Unflattened Photon Beams Shaped by Multileaf Collimator}

\author{
Kajaria A. ${ }^{*}$, Sharma N. ${ }^{1}$, Sharma Sh. ${ }^{1}$, Pradhan S. ${ }^{2}$, Mandal \\ A. ${ }^{2}$, Aggarwal L. M. ${ }^{2}$
}

\begin{abstract}
Introduction: This study investigates basic dosimetric properties of unflattened $6 \mathrm{MV}$ photon beam shaped by multileaf collimator and compares them with those of flattened beams.

Materials and Methods: Monte Carlo simulation model using BEAM code was developed for a $6 \mathrm{MV}$ photon beam based on Varian Clinic 600 unique performance linac operated with and without a flattening filter in beam line. Dosimetric features including lateral profiles, central axis depth dose, photon and electron spectra were calculated for flattened and unflattened cases, separately.

Results: An increase in absolute depth dose with a factor of more than 2.4 was observed for unflattened beam which was dependent on depth. PDDs values were found to be lower for unflattened beam for all field sizes. Significant decrease in calculated mlc leakage was observed when the flattening filter was removed from the beam line. The total scatter factor, $\mathrm{S}_{\mathrm{CP}}$ was found to show less variation with field sizes for unflattened beam indicating a decrease in head scatter. The beam profiles for unflattened case are found to have lower relative dose value in comparison with flattened beam near the field edge, and it falls off faster with distance.
\end{abstract}

Conclusion: Our study showed that increase in the dose rate and lower peripheral dose could be considered as realistic advantages for unflattened 6MV photon beams.

Keywords

Monte Carlo Simulation, Flattening Filter Free Beams, Multileaf Collimator

\section{Introduction}

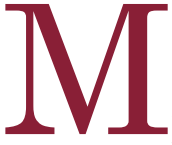

edical linear accelerators are equipped with a flattening filter (FF) as their integral part which is primarily designed to produce a flat beam profile by compensating for the non-uniformity of photon fluence across the field. Flattening filter is a major source of quality changes within the primary beam due to scattering and absorbing of primary photons, and it also decreases beam output noticeably. Introduction of new technologies such as intensity-modulated radiation therapy (IMRT) or intensity-modulated arc therapy have removed the necessity to have a flattened beam profile. In principle, the flattening filter can be removed, and the leaf sequences of mlc can be adjusted accordingly to produce desired fluence distributions similar to those of a beam with a flattening filter. Substantial reduction in head scatter as well as significant increases in dose rate can be achieved by the removal of flattening filter with its associated attenuation from the $\mathrm{x}$-ray
${ }^{1}$ School of Biomedical Engineering, Indian

Institute of Technology

(BHU), Varanasi, UP,

India

${ }^{2}$ Department of Radio-

therapy and Radiation

Medicine, Institute of

Medical Science (BHU),

Varanasi, UP, India

*Corresponding author: A. kajaria

School of Biomedical En-

gineering Indian Institute

of Technology (BHU),

Varanasi, UP, India

E-mail: akajaria.

rs.bme12@itbhu.ac.in

Received: 29 May 2016

Accepted: 15 June 2016 
beam path [1]. Thus flattening filter free (FFF) beams in radiotherapy have the advantage of shorter treatment delivery time and lower peripheral dose compared to conventional flattened beams. This is particularly vital for the treatment where large doses per fraction are advised, e.g., stereotactic ablative body radiotherapy [2,3] or where patient's motion might affect the efficacy of the delivery or both [4]. Many studies have been conducted using Monte Carlo (MC) method for analyzing the influence of linac head components on beam characteristics $[5,6,7]$. Thus, the effect of flattening filter on absolute absorbed dose, beam profiles and various energy spectra could be studied by this method [8]. In an MC study on flattening filter free beams, dose rates increase by a factor of 2.31(6 MV) and 5.45(18 MV) and out-of-field dose reductions were reported [9]. In a similar study, a significant improvement in out-of-field dose was reported for small field sizes [10]. The effects of mlc on beam characteristics for flattened and unflattened beam were also investigated in previous studies coating clear advantage of mlc over jaw to define treatment field [11]. Above-mentioned studies outline the possible profit of removing the flattening filter. It is therefore important to examine these properties for a typical modern accelerator such as Varian Clinic 600 unique performance. Our study reports on depth-dose dependencies, lateral profiles, total scatter factors, mlc leakage and various energy spectra in a conventional accelerator and a flattening filter-free system.

\section{Material and Methods}

\section{Beam-line Simulation Model for 6 MV Varian Linac}

Monte Carlo code system BEAMnrc [12, 13] is used to model Varian Clinic 600 unique performance in our study. To derive the best estimates for the mean energy and full width at half maximum (FWHM) of the electron beam incident on the target, Monte Carlo simulations for monoenergetic beams ranging from 5.5 to 6.2 MeV with FWHM varied from 0.15 to 0.25 $\mathrm{cm}$ were performed to find the best match with percentage depth dose (PDD) and profile measurements. A monoenergetic source with kinetic energy of the beam 5.7 MeV and FWHM for $\mathrm{X}$ and $\mathrm{Y}$ directions of $0.2 \mathrm{~cm}$ was found to give the best agreement with measured data. Geometry and materials used to build Monte Carlo simulation model of the linear accelerator were based on machine specifications as provided by the manufacturer Varian Medical Systems. The linac was structured in the following order: a target slab of tungsten and copper, primary collimator (tungsten), flattening filter, ion chamber, mirror, jaws (tungsten) and finally the option for 120- leaf Varian MillenniumTM Multileaf Collimator. All materials used in the Monte Carlo (MC) simulation were extracted from the 700 ICRU PEGS4 (pre-processor for Electron Gamma Shower) cross section data available in BEAMnrc, and met the specifications for the linac as provided by the manufacturer. Different stages of simulation for $6 \mathrm{MV}$ photon beam produced by Varian Linac using principal features of the BEAMnrc-DOSXYZnrc code [14, 15] are shown in Figure 1. In the simulation of the full accelerator unit, we have split the calculation into three steps in order to save time. In the first step which takes the most computing time, $1.5 \times 10^{8}$ initial histories are initiated and a monoenergetic electron beam source of kinetic energy of $5.7 \mathrm{MeV}$ with FWHM for the $\mathrm{X}$ and $\mathrm{Y}$ directions of $0.2 \mathrm{~cm}$ was incident on the target. The primary collimator, flattening filter and ion chamber are included in this step. The output of this step is a phase space file at plain one as show in Figure 1, having information of energy, position, direction, charge and history variable for every particle exiting downstream from the end of ion chamber. Since source and primary collimator have fixed openings, it is possible to use this phase space data for the simulation of different field sizes. Figure 1 lists the component module of 


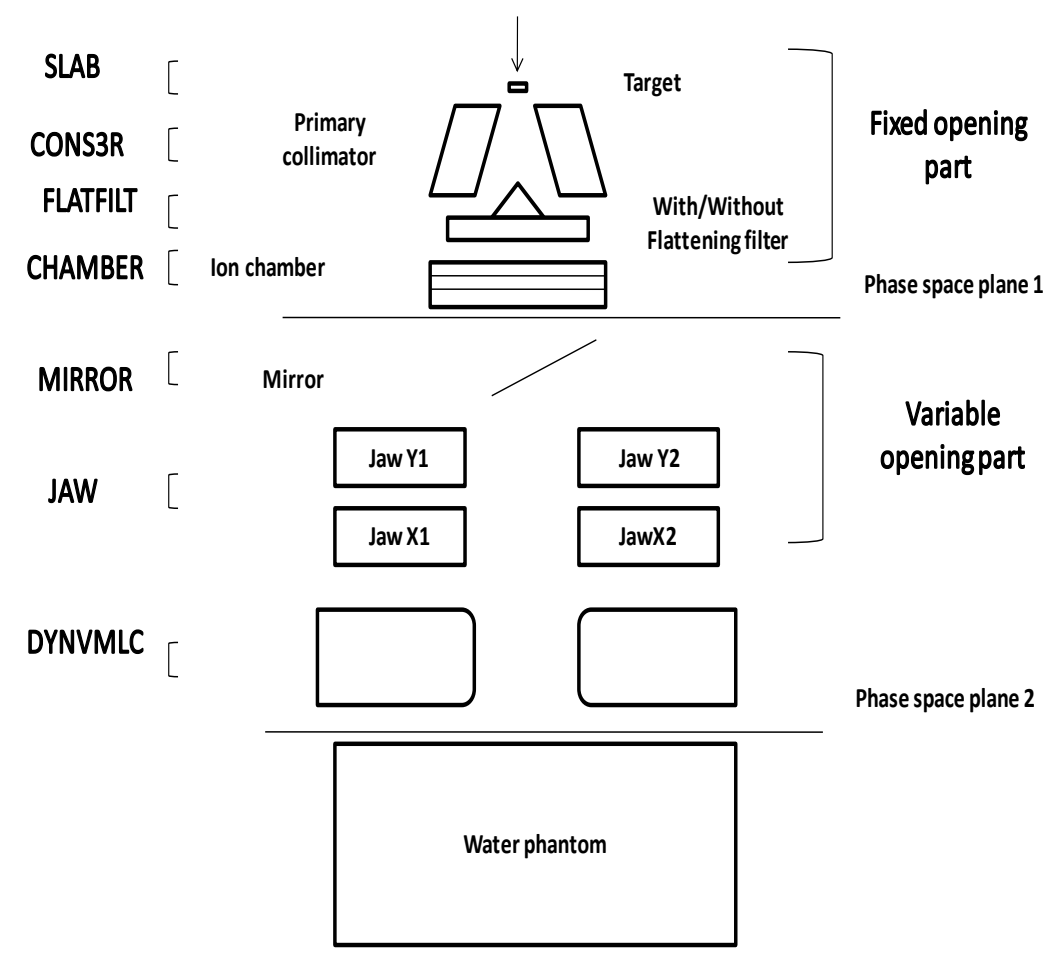

Figure 1: $6 \mathrm{MV}$ Varian linac simulation model separated into three parts, Treatment head fixed and variable opening part representing first and second step of simulation modelled using component module of BEAMnrc code and Dose Calculation inside water phantom using DOSXYZnrC code in third step.

BEAMnrc code used for modeling of fixed opening part of treatment head in the first step. This large set of particles produced in first step is used repeatedly as the input to the next step of simulation. The second step of calculation simulates the passage of the particles through the mirror, adjustable collimator, mlc and air slab to a plane at source to surface distance (SSD) $100 \mathrm{~cm}$ from target. We simulate different openings of jaw as well as mlc to get field sizes from $5 \times 5$ to $20 \times 20 \mathrm{~cm}^{2}$ at an SSD equal to $100 \mathrm{~cm}$. For the latter case in mlc defined field sizes the projected jaw setting was $5 \mathrm{~cm}$ larger than that of mlc. Figure 1 also lists the component module of BEAMnrc code used for modeling of variable opening part of treatment head in second step of simulation. The output of this step is a phase space file at plain two as shown in Figure 1, having information of energy, position, direction, charge and history variable for every particle reaching the plain at SSD $100 \mathrm{~cm}$ from target. The data analysis program BEAMDP [16] is used to analyze the phase space data files to extract various types of spectra of all particles reaching the plane at SSD $100 \mathrm{~cm}$. In the third step of the simulation, the phase space files for field sizes of $5 \times 5$ to $20 \times 20 \mathrm{~cm}^{2}$ at an SSD of $100 \mathrm{~cm}$ which are obtained at the end of second step are reused by the DOSXYZnrc code as an input for dose calculations in a water phantom as shown in Figure 1. We transport particles through a water phantom of dimension $30 \times 30 \times 30 \mathrm{~cm}^{3}$ with voxels size of $0.25 \times 0.25 \times 0.25 \mathrm{~cm}^{3}$. In the simulation of "unfiltered" $6 \mathrm{MV}$ photon beam, all three steps of simulation are the same expect in first step where the flattening filter is being removed from the beam line. A comprehen- 
sive set of dosimetric data for $6 \mathrm{MV}$ filtered photon beams where acquired using a threedimensional (3D) phantom, Blue phontom ${ }^{2}$ IBA Dosimetry GmbH and OmniPro-Accept 7 data acquisition software. All the measurements were performed with a Scanditronix/ Wellhofer compact ionization chamber $\mathrm{CC} 13$, in the water phantom.

\section{Results}

\section{Validation of Monte Carlo Simula- tion Model}

Depth-dose curves for filtered 6MV photon beam for field size $5 \times 5$ to $20 \times 20 \mathrm{~cm}^{2}$ were calculated in an on axis cylinder of radius $1 \mathrm{~cm}$ using Monte Carlo simulation (MC) and compared with measured data for the validation of simulation model. The calculated central axis depth-dose curves were normalized to unity at the depth, $\mathrm{d}_{\max }$, of the maximum dose deposition and the $\mathrm{D}_{\max }$. Both results measured and calculated, could then be compared with respect to the relative value of the maximum dose $\mathrm{D}_{\max }$ and the corresponding depth $\mathrm{d}_{\max }$. Figure 2 shows the comparison between the calculated depth-dose distributions and measurements of three different field sizes. The comparison shows that the calculated and measured data agree within $1 \%$ of local relative dose, and $1 \mathrm{~mm}$ in depth at all depths and field sizes which are summarized in Table 1.

Lateral beam profiles for the filtered $6 \mathrm{MV}$ photon beam were also simulated for $5 \times 5$ to $20 \times 20 \mathrm{~cm}^{2}$ field sizes at $1.5,5$ and $10 \mathrm{~cm}$ depths. The measured and calculated lateral dose profiles were normalized to unity on the central axis for comparison. Figure 3 shows the comparison of Monte Carlo calculations to measured data for a field size of $20 \times 20$, $10 \times 10$ and $5 \times 5 \mathrm{~cm}^{2}$ at depth of $10 \mathrm{~cm}$. The lateral field size at the $50 \%$ dose level $\left(\mathrm{X}_{50}\right)$ and penumbra widths, $\mathrm{P}_{90-10}$ and $\mathrm{P}_{80-20}$ (calculated from the $90 \%$ level to the $10 \%$ level

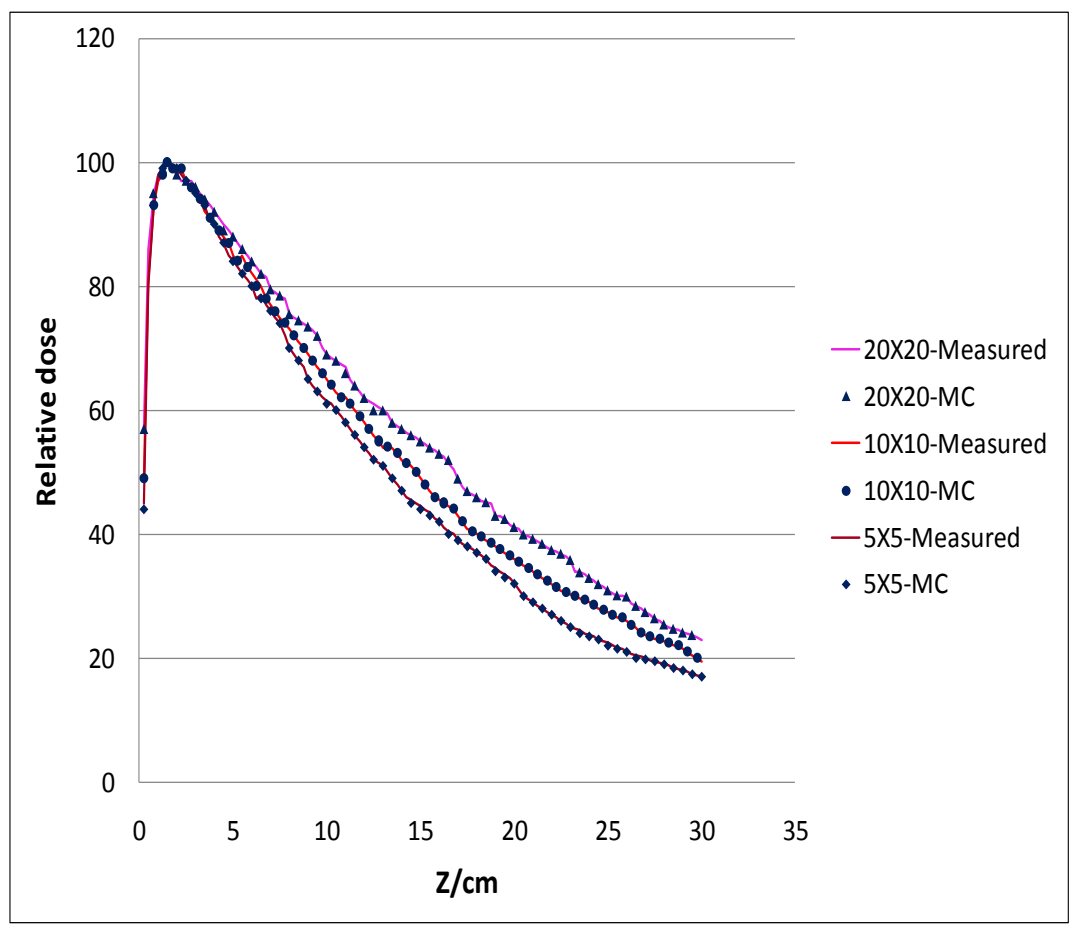

Figure 2: Comparison of measured and calculated depth doses curves of $6 \mathrm{MV}$ photon beam for $20 \times 20,10 \times 10$ and $5 \times 5 \mathrm{~cm}^{2}$ field sizes. 
Table 1: Comparison of calculated and measured central-axis depth-dose profiles at various Field sizes. A denotes the field size, $d_{\max }(\mathrm{cm})$ denotes the location of the maximum Dose, and $\Delta \mathrm{D}_{\max }$ is the relative dose difference between the measurement and the Calculations at $\mathrm{d}_{\max }$

\begin{tabular}{cccc}
$\mathbf{A} / \mathbf{c m}^{2}$ & $\mathbf{d}_{\max }$ (simulated) & $\mathbf{d}_{\max }$ (measured) & $\boldsymbol{\Delta D}_{\max }$ \\
\hline $5 \times 5$ & 1.5 & 1.56 & 0.2 \\
\hline $10 \times 10$ & 1.5 & 1.52 & 0.17 \\
\hline $15 \times 15$ & 1.48 & 1.5 & 0.13 \\
\hline $20 \times 20$ & 1.38 & 1.40 & 0.10
\end{tabular}

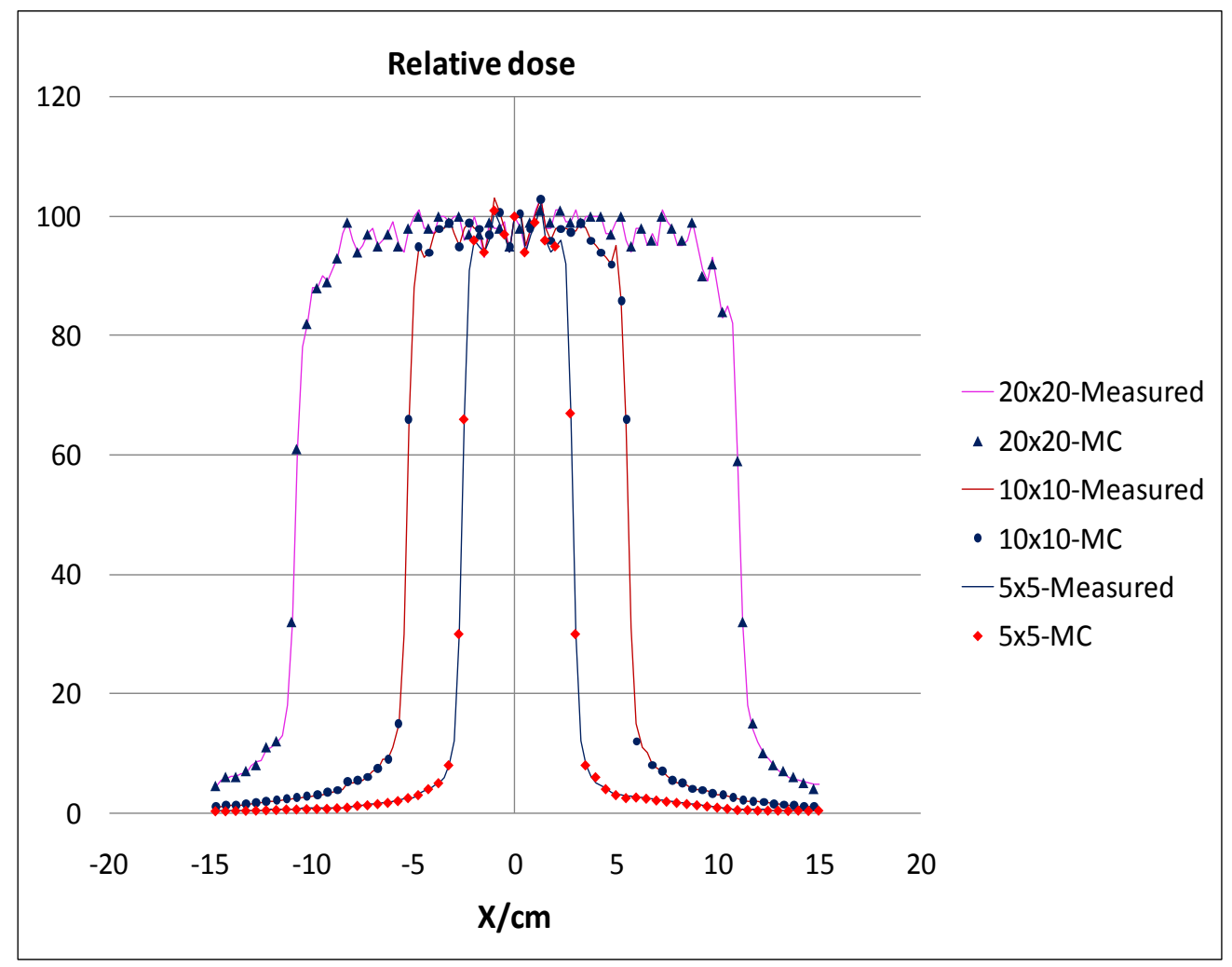

Figure 3: Comparison of measured and calculated beam profiles of the $6 \mathrm{MV}$ photon beam at a Depth of $10 \mathrm{~cm}$ for $20 \times 20,10 \times 10$ and $5 \times 5 \mathrm{~cm}^{2}$ field sizes. 
and from $80 \%$ to $20 \%$ ) where calculated using Monte Carlo simulation and the results of the comparisons are summarized in Table 2. The differences between the measurement and the simulations results in lateral field size at the $50 \%$ dose level, $\mathrm{X}_{50}$, was found to be less than $1 \mathrm{~mm}$.

\section{Comparison of Flattened and Unflat- tened Beam Characteristics \\ Profile Comparison}

Beam profiles for different field sizes were calculated at 1.5,5 and $10 \mathrm{~cm}$ depth for both cases with/without flattening filter in a water phantom. The lateral profile for $20 \times 20 \mathrm{~cm}^{2}$ and $10 \times 10 \mathrm{~cm}^{2}$ field sizes at a depth of $10 \mathrm{~cm}$ are compared for two cases as shown in Figures 4 and 5. For this comparison of unflattened beam with flattened one, the flat profile is normalized to 1 on the central axis, and the nonflat profile is normalized by dose $\left(D_{n}\right)$ which is calculated using this formula:

$$
D_{n}=\left(\frac{D_{u}}{D_{f}}\right) * D_{C A X}
$$

Where $D_{u}$ is the dose at the inflection point of penumbra region of the unflattened beam, $D_{f}$ is the dose at the inflection point of the flattened profile and $D_{C A X}$ is the dose on the central axis of the flattened beam [11]. It is observed in our study that the beam profiles for unflattened case to have relative dose value lower than the flattened beam are close to the measured field size edge. However, the amount of reduction in dose was less for mlc shaped in comparison to jaw shaped unflattened beam. For field size $20 \times 20 \mathrm{~cm}^{2}$ measured at $9 \mathrm{~cm}$ off axis distance reduction in relative dose for mlc and jaw shaped unflattened beam was found to be $12 \%$ and $15 \%$, respectively. For field size $10 \times 10 \mathrm{~cm}^{2}$ measured at $4 \mathrm{~cm}$ off axis distance reduction in relative dose for mlc shaped unflattened beam was found to be $8 \%$, respectively.

The dose in near field edge region for small field size shaped by mlc was investigated in
Table 2: Comparison of measured and calculated lateral dose profiles at $10 \mathrm{~cm}$ depth. A Denotes the field Size, $\Delta X_{50}(\mathrm{~mm})$ is the lateral difference measured at the $50 \%$ Dose point in the Penumbra, and $\Delta \mathrm{P}_{90-10}(\mathrm{~mm})$ as well as $\Delta \mathrm{P}_{80-20}(\mathrm{~mm})$ describe the Difference in width of the penumbra measured from the $90 \%$ point to $10 \%$ dose Point and from $80 \%$ to $20 \%$ Dose point respectively.

\begin{tabular}{cccc}
$\mathbf{A} / \mathbf{c m}^{\mathbf{2}}$ & $\boldsymbol{\Delta} \mathbf{X}_{\mathbf{5 0}}$ & $\boldsymbol{\Delta} \mathbf{P}_{\mathbf{8 0 - 2 0}}$ & $\boldsymbol{\Delta} \mathbf{P}_{\mathbf{9 0 - 1 0}}$ \\
\hline $5 \times 5$ & 0.10 & 1.5 & 0.8 \\
\hline $10 \times 10$ & 0.50 & 1.52 & 1.0 \\
\hline $15 \times 15$ & 0.40 & 1.2 & 2.0 \\
\hline $20 \times 20$ & 0.50 & 1.0 & 2.2
\end{tabular}

our study for unflattened beam and compared with that of the flattened beam. Figure 6 shows the calculated flattened and unflattened beam profiles for a small field size $\left(5 \times 5 \mathrm{~cm}^{2}\right)$ at a depth of $5 \mathrm{~cm}$. The dose at $2 \mathrm{~cm}$ off-axis distance is lower in unflattened beams by $5 \%$ and it tends to decrease faster with increasing off axis distance than in flattened beams. Faster lateral dose fall-off outside the treatment field will result in lower doses to surround normal tissues.

\section{Analysis of Spectra \\ Photon Energy Fluences Spectra}

Figure 7 shows photon energy fluences as a function of off axis distance calculated for $20 \times 20 \mathrm{~cm}^{2}$ field size. Photon emerging from target passes through the components of the collimating system on their way to the scoring plain at an SSD $100 \mathrm{~cm}$. Scoring plain is an annular region around the central axis with radius $0<\mathrm{r}<15 \mathrm{~cm}$. The annular region has been divided into equal interval (bin) of 0.5 $\mathrm{cm}$. The number of photon within each bin crossing the scoring plain is being recorded for with/without flattening filter case separately. The precision of calculated photon fluences spectra for all field sizes used in the dose 


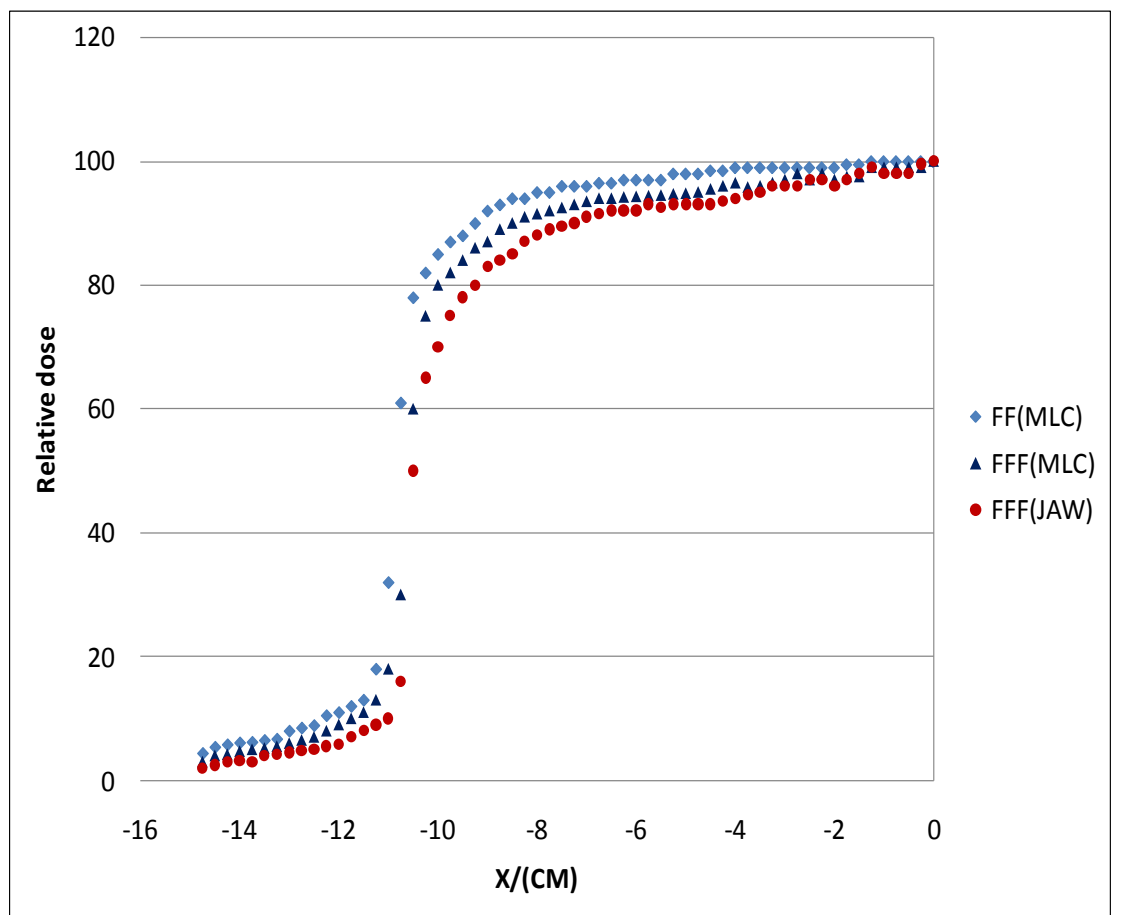

Figure 4: Comparison of lateral profile for $6 \mathrm{MV}$ photon beam at $10 \mathrm{~cm}$ depth for field size $20 \times 20$ $\mathrm{cm}^{2}$. FF (MLC) denotes flattened beam shaped by mlc, FFF (MLC) and FFF (JAW) denotes unflattened beam shaped by mlc and jaw, respectively.

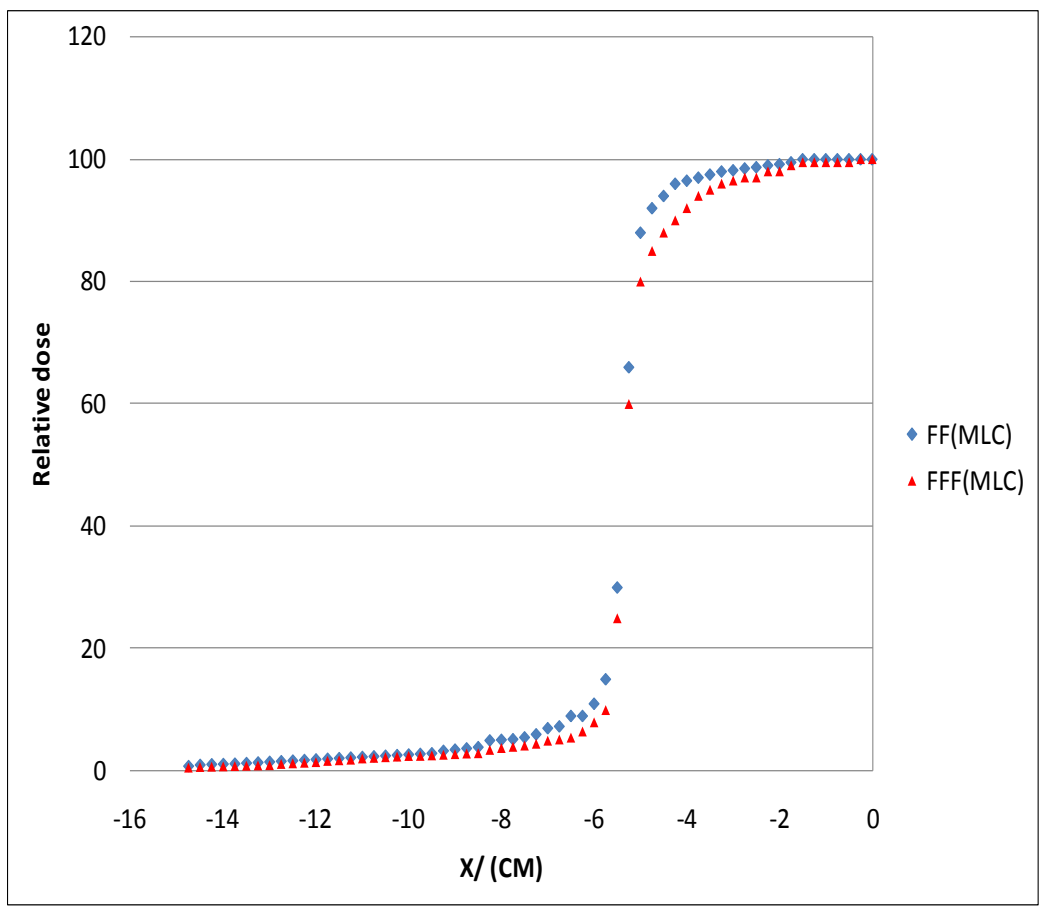

Figure 5: Comparison of lateral profile for $6 \mathrm{MV}$ photon beam with/without a flattening filter in Beam line Shaped by mlc at depth $10 \mathrm{~cm}$ for field size $10 \times 10 \mathrm{~cm}^{2}$. FF and FFF denotes calculation with/without a flattening filter in beam line. 


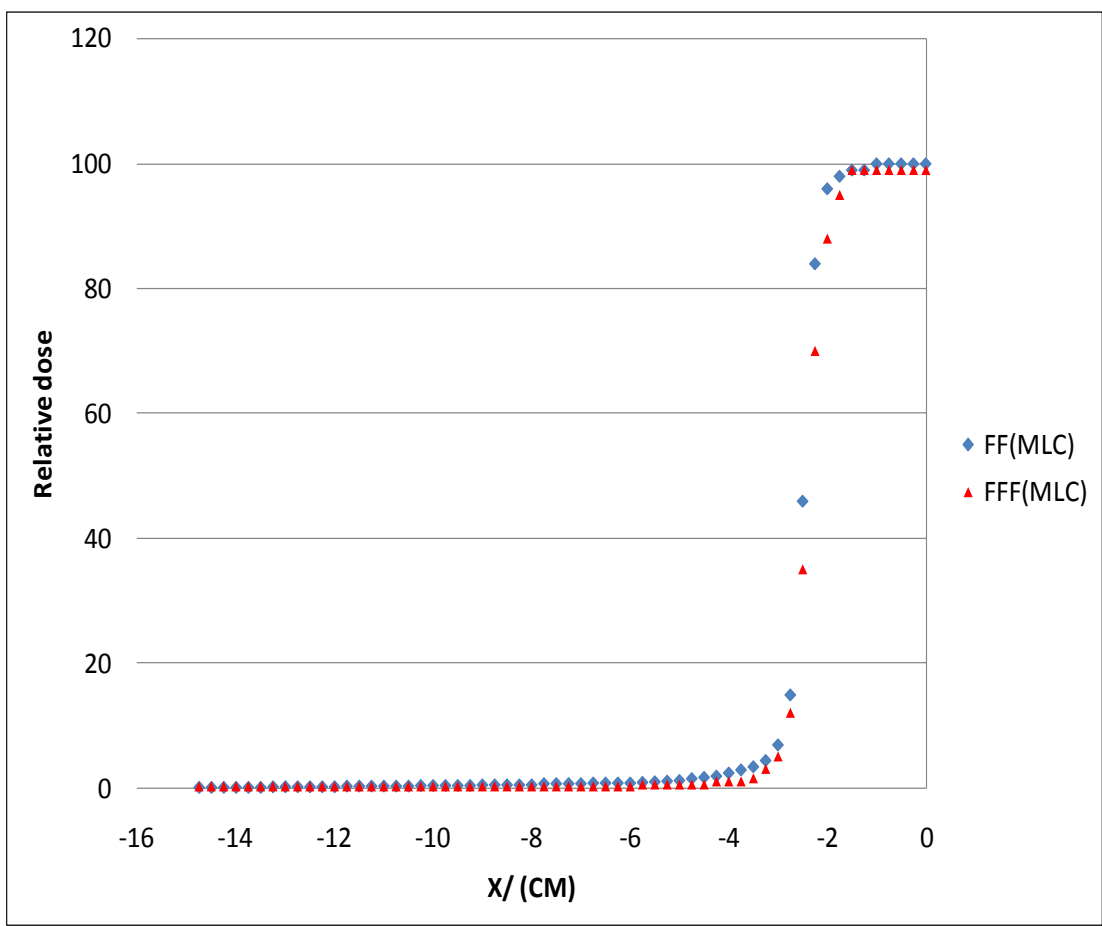

Figure 6: Comparison of lateral dose profiles Shaped by mlc for a $5 \times 5 \mathrm{~cm}^{2}$ field size at a depth of $5 \mathrm{~cm}$. FF and FFF denotes calculation with/without a flattening filter in beam line.

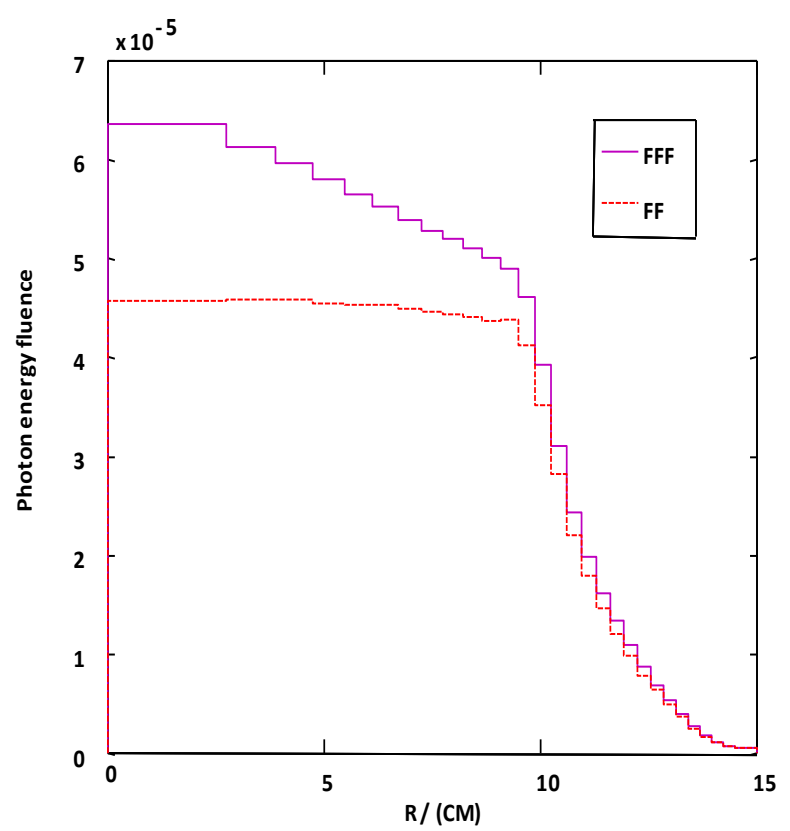

Figure 7: Photon energy fluences per initial electron on the target, at the top of the water phantom as a function of off axis distance for $20 \times 20 \mathrm{~cm}^{2}$ field size calculated for with/ without a flattening filter in beam line. calculations is high and uncertainty in each $0.5 \mathrm{~cm}$ wide bin is usually between 1 to $5 \%$, except for the high-energy end of the spectra. There is a noticeable increase observed in the photon energy fluence when the flattening filter is removed from the beam line.

Average Energy Distribution

Figure 8 shows the calculated photon average energies distribution at $100 \mathrm{~cm} \mathrm{SSD} \mathrm{for}$ $20 \times 20 \mathrm{~cm}^{2}$ field size as a function of off axis distance for with/without flattening filter case. From the above distribution beam hardening effect produced by the flattening filter [8] for the filtered beam can be verified as we find that the mean photon energy for flattened beam to have a value at central axis $1.5 \mathrm{MeV}$ and decreases to $1.12 \mathrm{MeV}$ at off axis distance of 20 $\mathrm{cm}$. In cases of unflattened beam, the mean energy of spectra did not change significantly with increasing off axis distance, and it respectively decreased from $1.2 \mathrm{MeV}$ on central axis to $1.10 \mathrm{MeV}$ at $20 \mathrm{~cm}$ off axis distance. 


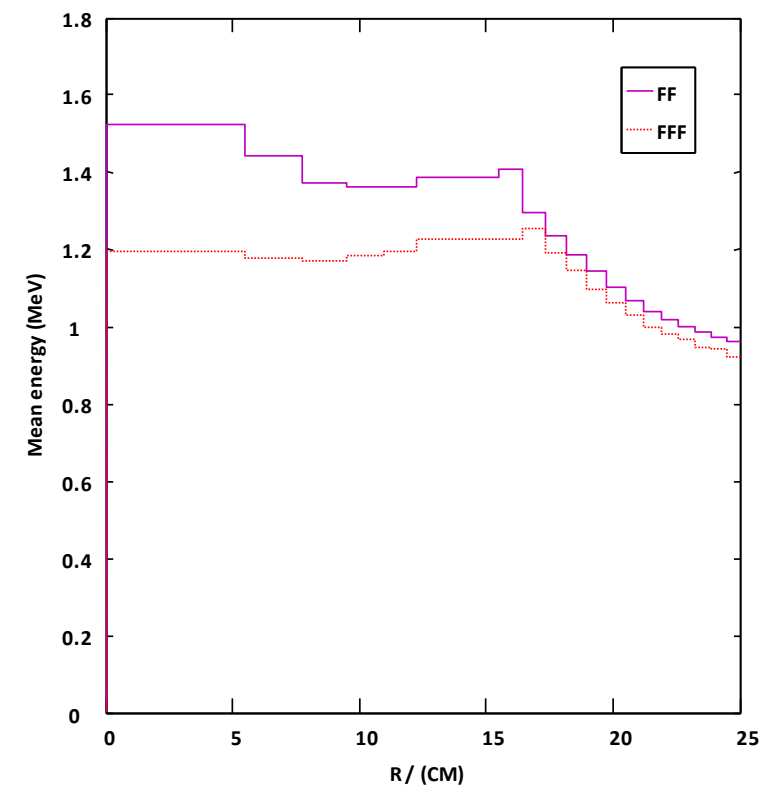

Figure 8: Photon average energy distribution of the filtered and unfiltered 6-MV beams as a function of the off axis distance for $20 \times 20$ $\mathrm{cm}^{2}$ field size.

\section{Contaminant Electron Energy Fluence Spectra}

Figure 9 shows the calculated electron energy fluence spectra for contaminant electrons calculated as a function of off axis distance for $20 \times 20 \mathrm{~cm}^{2}$ field size at $100 \mathrm{~cm}$ SSD for with/ without flattening filter case separately. Number of electron reaching the phantom surface is found to increase with removing the flattening filter from the beam line. However, the difference at near center is higher and tends to decrease as the off axis distances increase between the two cases. Monte Carlo calculation demonstrated that the electron fluence at the center for a $6 \mathrm{MV}$ flattening filter free beam with the same electron beam hitting the target was found to be 1.2 times greater than its value for with flattening filter case for field size $20 \times 20 \mathrm{~cm}^{2}$. This increase in electron energy fluence indicates a potential risk of delivering a high skin dose to the patient and also the risk of placing ion chamber used for the measurement outside the range of its reliable operation.

Depth Dose Analysis

Absolute Dose: For comparison purposes, absolute absorbed doses per initial electron on target were calculated for flattened and unflattened beam at two reference depths of 1.5 and $10 \mathrm{~cm}$. The ratio of absolute depth doses for flattening filter free to standard flattened beams were calculated and are presented in Table 3. A significant increase in absorbed dose was observe by removing flattening filter, indicating an increased in dose rate for unflattened beam. However, the increase in dose rate decreases with increasing depth.

Percentage Depth-Dose Characteristics: Absolute depth dose values were used to calculate percentage depth-dose characteristic (PDD) curves. It can be seen from Figure 10 that unflattened beam shows slightly lower PDDs values in comparison with the standard beam for all field sizes. Difference in PDDs

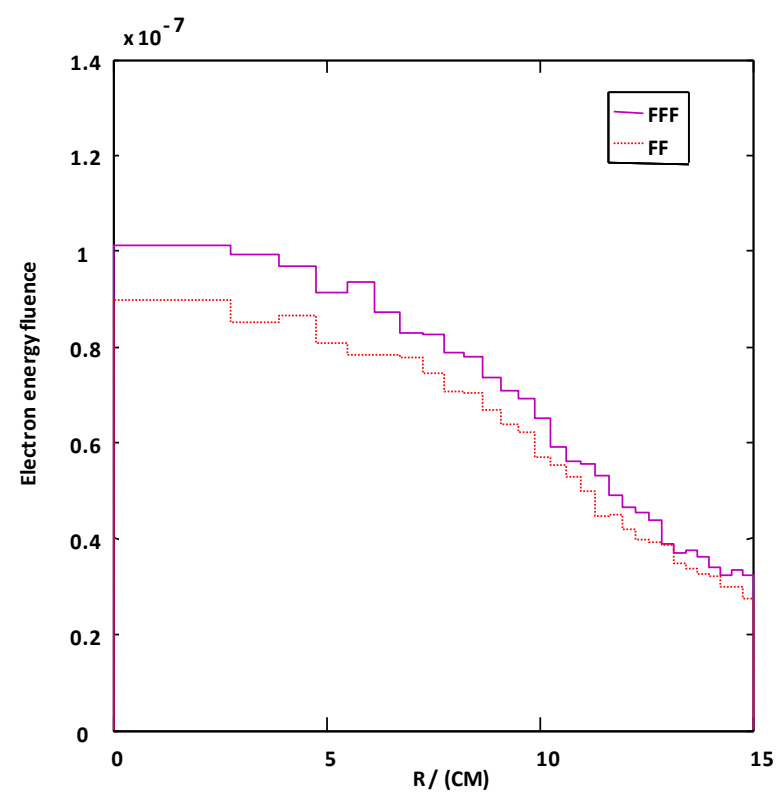

Figure 9: Electron energy fluences per initial electron on target, at the top of the water phantom as a function of off axis distance for $20 \times 20 \mathrm{~cm}^{2}$ field size calculated for with/ without a flattening filter in beam line. 
Table 3: Ratios of absolute depth doses for flattening filter free to flattened beams at two reference depths for different field sizes. A denotes the field size; $d$ denotes the depth inside water phantom. Absorbed dose calculated without the flattening filter in the beam line is denoted as $D_{\text {FFF }}$ (flattening filter free) and with filter in beam line is denoted as $D_{\mathrm{FF}}$.

$\mathbf{A} / \mathbf{c m}^{2}\left(\frac{D_{F F F}}{D_{F F}}\right)$ At $\mathbf{d}=\mathbf{1 . 5}\left(\frac{D_{F F F}}{D_{F F}}\right)$ At $\mathbf{d}=\mathbf{1 0}$

\begin{tabular}{ccc}
\hline $5 \times 5$ & 2.49 & 2.42 \\
\hline $10 \times 10$ & 2.47 & 2.45 \\
\hline $20 \times 20$ & 2.44 & 2.40
\end{tabular}

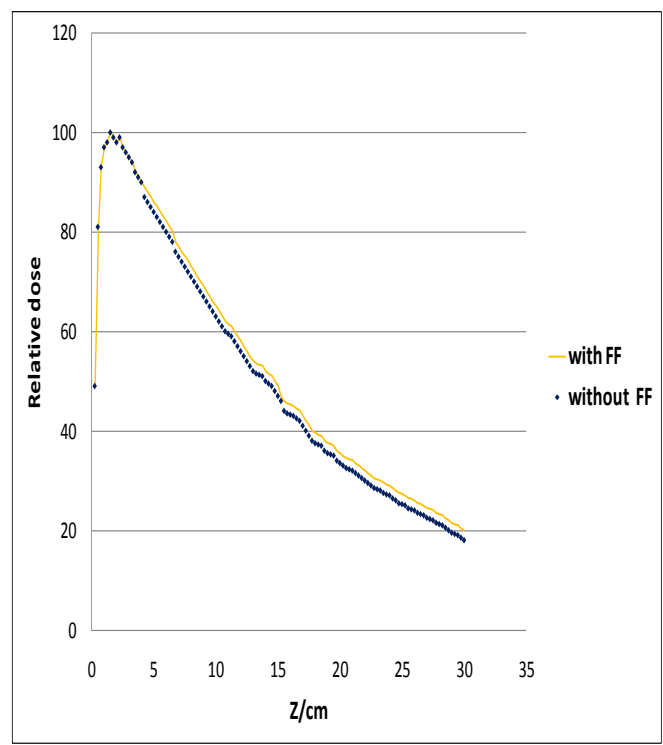

(a) between the two cases is evident at deeper depths and increasedswith depth for $10 \times 10$ and $20 \times 20 \mathrm{~cm}^{2}$ field sizes. This difference is validated by calculating two parameters which are reported in Table 4, namely, the relative dose at a depth of 10 and $20 \mathrm{~cm}\left(\mathrm{D}_{10}, \mathrm{D}_{20}\right)$.

\section{MLC Linkage}

MLC leakage is an important parameter needed for the commissioning of a treatmentplanning system. We calculated the MLC leakage as a function of field size for the unflattened profile in our study as presented in Table 5. MLC leakage represents the dose on the central beam axis with MLC blocked fields normalized by the dose of open fields of the same field size at $1.5 \mathrm{~cm}$ depth for SSD 100 $\mathrm{cm}$. Open MLC fields are defined as MLC leaves are withdrawn underneath the jaws as not to intercept the beam, the field size is defined by the treatment jaws. MLC blocked fields define a field in which the MLC leaves

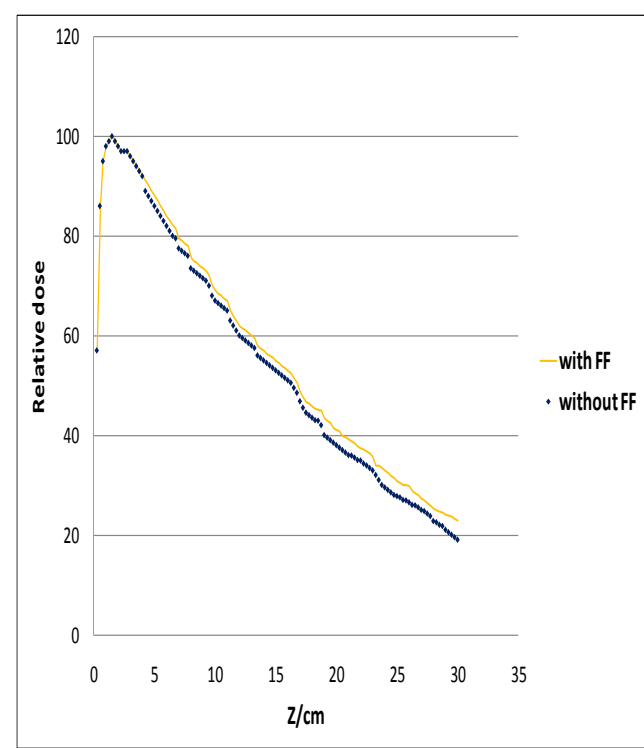

(b)

Figure 10: Comparison of relative depth dose curves calculated for with and without flattening filter for 6MV photon beams for different field sizes: (a) $10 \times 10 \mathrm{~cm}^{2}$ (b) $20 \times 20 \mathrm{~cm}^{2}$ 
Table 4: Comparison of relative depth doses for flattening filter free to standard flattened beams at two reference depths for different Field sizes. A denotes the field size; $D_{10}$ and $\mathrm{D}_{20}$ denote relative depth dose at 10 and 20 $\mathrm{cm}$ depth.

\begin{tabular}{|c|c|c|c|c|}
\hline \multirow[b]{2}{*}{$\mathrm{A} / \mathrm{cm}^{2}$} & \multicolumn{2}{|c|}{$D_{10}$} & \multicolumn{2}{|c|}{$D_{20}$} \\
\hline & $\begin{array}{c}\text { With } \\
\text { FF }\end{array}$ & $\begin{array}{c}\text { Without } \\
\text { FF }\end{array}$ & $\begin{array}{c}\text { With } \\
\text { FF }\end{array}$ & $\begin{array}{c}\text { Without } \\
\text { FF }\end{array}$ \\
\hline $5 \times 5$ & 62.43 & 59.40 & 33.26 & 30.84 \\
\hline $10 \times 10$ & 66.70 & 63.80 & 37.80 & 34.34 \\
\hline $20 \times 20$ & 71.65 & 68.96 & 40.98 & 37.55 \\
\hline
\end{tabular}

are configured to fully block the open field produced by the jaw. To ensure that the jaws blocked the rounded tips of the leaves completely in MLC blocked fields the leaves of MLC were positioned asymmetrically with respect to the central axis, and there projected offset at isocenter was $8.0 \mathrm{~cm}$.

\section{Scatter Function}

The total scatter factor, SCP is defined as 'the dose rate at a reference depth for a given field size divided by the dose rate at the same point and depth for the reference field size $(10 \times 10$ $\mathrm{cm}^{2}$ ). It was measured at SSD $=100 \mathrm{~cm}$ and
Table 5: Calculated MLC leakage for $6 \mathrm{MV}$ photon beam delivered with or without flattening Filter in beam line for different field sizes. Calculations were made at $1.5 \mathrm{~cm}$ depth and SSD $100 \mathrm{~cm}$.

\begin{tabular}{ccc} 
Field & \multicolumn{2}{c}{ MLC LINKAGE } \\
\cline { 2 - 3 } size & $\begin{array}{c}\text { With flattening } \\
\text { filter }\end{array}$ & $\begin{array}{c}\text { Without flattening } \\
\text { filter }\end{array}$ \\
\hline $5 \times 5$ & - & 1.10 \\
\hline $10 \times 10$ & 1.40 & 1.23 \\
\hline $20 \times 20$ & - & 1.32
\end{tabular}

a depth equal to $d_{\max }$ of a $10 \times 10 \mathrm{~cm}^{2}$ field for different field sizes. The data for with/without flattening filter case are presented in Table 6 .

The unflattened beams are found to have less value of SCP for lager field sizes in comparison wuth flattened beams indicating a reduced head scatter in unflattened beams compared with the standard flattened beam.

\section{Discussion}

Flattening filter is a main source of absorption of majority portion of primary photons particularly of those which are close to cen-

Table 6: Total scatter factor Scp of $6 \mathrm{MV}$ photon beams measured for with / without a flattening filter cases. The Scp was measured at SSD $=100 \mathrm{~cm}$, and at the depth of maximum dose $d_{\max }$ of a $10 \times 10 \mathrm{~cm}^{2}$ field size.

\begin{tabular}{cccc} 
Field size & $\begin{array}{c}\text { Scp (MLC shaped with } \\
\text { flattening filter) }\end{array}$ & $\begin{array}{c}\text { Scp (JAW shaped without } \\
\text { flattening filter) }\end{array}$ & $\begin{array}{c}\text { Scp (MLC shaped without } \\
\text { flattening filter) }\end{array}$ \\
\hline $5 \times 5$ & 0.967 & 0.97 & 0.98 \\
\hline $10 \times 10$ & 1 & 1 & 1 \\
\hline $15 \times 15$ & 1.021 & 1.012 & 1.010 \\
\hline $20 \times 20$ & 1.054 & 1.027 & 1.018
\end{tabular}


tral axis of beam, thus removing filter from the beam line should result in considerable increase in dose rate, and therefore a decrease in beam-on time should be achieved when radiation treatment is delivered. The ratios of absolute depth doses calculated in our study for flattening filter free to standard flattened beams for field size $10 \times 10 \mathrm{~cm}^{2}$, at $10 \mathrm{~cm}$ depth for an SSD equal to $100 \mathrm{~cm}$ was found to be 2.45 signifying the potential higher dose rate delivered by the unflattened beam. Unflattened beam is found to have slightly lower PDDs value compared to the standard beam for all field sizes. Difference in the PDDs of flattened and unflattened beams are apparent at deeper depths and increase with depth for all field sizes. The photon energy fluence and average energy distribution as a function of off axis distance for flattened and unflattened beams are being calculated in our study. It was observed that the fluence of photon on central axis increased nearly two times with removing flattening filter, and with increasing off axis distance this difference reduced. The average energy of photon spectrum on central axis decreased from 1.5 to $1.2 \mathrm{MeV}$ for $20 \times 20$ $\mathrm{cm}^{2}$ field size at $100 \mathrm{~cm}$ SSD with removing flattening filter. The average energy of photon for flattened beam was found to decrease with increasing off axis distance from $1.5 \mathrm{MeV}$ on central axis to $1.12 \mathrm{MeV}$ at $20 \mathrm{~cm}$ off axis distance for $20 \times 20 \mathrm{~cm}^{2}$ field size. Whereas for flattening filter free beam, the mean energy of spectra did not change significantly with increasing off axis distance, and it respectively decreased from 1.2 $\mathrm{MeV}$ on central axis to $1.1 \mathrm{MeV}$ at $20 \mathrm{~cm}$ off axis distance for $20 \times 20$ $\mathrm{cm}^{2}$ field size. The differential attenuation produced by flattening filter with increasing off axis distance from central axis of beam is the possible explanation for this behavior. As the central thick part of flattening filter attenuates more low energy photons, and as off axis distance increases, more low energy photons are allowed to penetrate the thin lateral part of the flattening filter contributing to the photon en- ergy fluence; thus the mean energy of spectra decreases significantly with increasing distance for the flattened beam. In our study, we have calculated the mlc leakage for both cases with or without flattening filter in the beam line. It was observed that there is a considerable decrease in mlc leakage when the flattening filter was removed from the beam line, as for $10 \times 10 \mathrm{~cm}^{2}$ field size, its value was 1.4 which decreases to 1.23 with filter removed from the beam line. The average energy difference on the central axis is considered to be the major reason for this decrease. As the filter is removed from the beam line, the average energy of photon beam decreases causing more attenuation of the photon beam by mlc. The total scatter factor, SCP for the unflattened and flattened beam has been investigated in our study. It can be seen from the data that the flattening filter free beam SCP increases more slowly with increasing field size than that of the flattened beam. In addition, the amount of variation in SCP was even less for mlc shaped in comparison with jaw shaped unflattened beam; this is due to the forward-peaked profile of unflattened beam, which produces less SCP because of the reduced off-axis intensity. The flattening filter free beam has greatly reduced fluence off axis; hence, less secondary head scatter is created, which is directed towards the central axis. Due to this reason, as the measured field size increases, the expected increase in SCP for flattening filter free beams is not seen which is found with the flattened ones. Our study showed that flattened and unflattened beams are comparable within a few centimetres from the central axis; therefore, unflattened beams are unlikely to present a problem for treatments with small fields. Moreover, the treatments can also profit from an increased dose rate. The beam profiles for unflattened cases are found to have lower relative dose value in comparison with flattened beam close to the field edge. The key reason for this behavior is that the flattening filter elevates relative fluence of primary photons 
propagating off-axis and reduces head scatter present in unflattened beams. The near field edge dose calculated without the flattening filter is found to be smaller for small field sizes when compared with the flattened beams; it falls off faster with distance. This means that an improved sparing of normal tissues close to small treatment fields can be achieved.

\section{Conclusion}

We developed a Monte Carlo simulation model for a $6 \mathrm{MV}$ photon beam based on Varian Clinic 600 unique performance linac and calculated basic dosimetric features which were benchmarked against measurement. It was then used to investigate the basic dosimetric properties of photon beams generated by the accelerator with the flattening filter removed. Comparison of dosimetric features of flattened and unflattened beams revealed that beam profiles for unflattened cases were found to have faster rate of decline and lower dose value. Mlc leakage calculated for both cases showed a considerable decrease for unflattened beam which is due to the differences in average energy on the central axis for the two cases. The total scatter factor, SCP less variation with field sizes indicate that removing the filter from the beam line could significantly reduce the amount of head scatter photons and therefore doses to normal tissues and organs.

\section{Acknowledgment}

The authors of this article wish to thank Varian Medical Systems for providing them with the specifications needed for linac simulations.

\section{Conflict of Interest}

None

\section{References}

1. Fu W, Dai J, Hu Y, Han D, Song Y. Delivery time comparison for intensity-modulated radiation therapy with/without flattening filter: a planning study. Phys Med Biol. 2004;49:1535-47. doi.org/10.1088/0031-9155/49/8/011. PubMed PMID: 15152690.
2. Gillies BA, O'Brien PF, McVittie R, McParland C, Easton $\mathrm{H}$. Engineering modifications for dynamic stereotactically assisted radiotherapy. Med Phys. 1993;20:1491-5. doi.org/10.1118/1.597112. PubMed PMID: 8289733.

3. O'Brien PF, Gillies BA, Schwartz M, Young C, Davey P. Radiosurgery with unflattened 6-MV photon beams. Med Phys. 1991;18:519-21. doi. org/10.1118/1.596656. PubMed PMID: 1908047.

4. Jeraj R, Mackie TR, Balog J, Olivera G, Pearson $D$, Kapatoes J, et al. Radiation characteristics of helical tomotherapy. Med Phys. 2004;31:396404. doi.org/10.1118/1.1639148. PubMed PMID: 15000626.

5. Verhaegen F, Seuntjens J. Monte Carlo modelling of external radiotherapy photon beams. Phys Med Biol. 2003;48:R107-64. doi.org/10.1088/00319155/48/21/R01. PubMed PMID: 14653555.

6. Sheikh-Bagheri D, Rogers DW. Monte Carlo calculation of nine megavoltage photon beam spectra using the BEAM code. Med Phys. 2002;29:391402. doi.org/10.1118/1.1445413. PubMed PMID: 11930914.

7. Mesbahi A, Reilly AJ, Thwaites DI. Development and commissioning of a Monte Carlo photon beam model for Varian Clinac 2100EX linear accelerator. Appl Radiat Isot. 2006;64:656-62. doi. org/10.1016/j.apradiso.2005.12.012. PubMed PMID: 16455264.

8. Lee PC. Monte Carlo simulations of the differential beam hardening effect of a flattening filter on a therapeutic x-ray beam. Med Phys. 1997;24:1485-9. doi.org/10.1118/1.598037. PubMed PMID: 9304577.

9. Vassiliev ON, Titt U, Kry SF, Ponisch F, Gillin MT, Mohan R. Monte Carlo study of photon fields from a flattening filter-free clinical accelerator. Med Phys. 2006;33:820-7. doi.org/10.1118/1.2174720. PubMed PMID: 16696457.

10. Titt U, Vassiliev ON, Ponisch F, Dong L, Liu H, Mohan R. A flattening filter free photon treatment concept evaluation with Monte Carlo. Med Phys. 2006;33:1595-602. doi.org/10.1118/1.2198327. PubMed PMID: 16872067.

11. Ponisch F, Titt U, Vassiliev ON, Kry SF, Mohan R. Properties of unflattened photon beams shaped by a multileaf collimator. Med Phys. 2006;33:1738-46. doi.org/10.1118/1.2201149. PubMed PMID: 16872081.

12. Rogers DW, Faddegon BA, Ding GX, Ma CM, We J, Mackie TR. BEAM: a Monte Carlo code to 
simulate radiotherapy treatment units. Med Phys. 1995;22:503-24. doi.org/10.1118/1.597552. PubMed PMID: 7643786.

13. Rogers D, Ma C, Walters B, Ding G, Sheikh-Bagheri D, Zhang G. BEAMnrc Users Manual National Research Council of Canada. NRCC Report PIRS0509 (A) revK. 2002.

14. Kawrakow I, Walters BR. Efficient photon beam dose calculations using DOSXYZnrC with BEAMnrc. Med Phys. 2006;33:3046-56. doi.org/10.1118/1.2219778. PubMed PMID: 16964882.

15. Walters B, Kawrakow I, Rogers D. DOSXYZnrC users manual. NRC Report PIRS. 2005;794.

16. Ma C, Rogers D. BEAMDP as a General Purpose Utility National Research Council of Canada Report no. PIRS-0509 (E)(NRCC, Ottawa, Canada). 1998. 